\title{
A Divide-and-Conquer Approach with Adaptive Modulation to CDMA systems
}

\author{
Satyam Dwivedi, T. S. Vedavathy, A. P. Shivaprasad \\ Authors are in Department of Electrical Communication Engineering, Indian Institute of Science, Bangalore, 560012, INDIA \\ email: satyam@protocol.ece.iisc.ernet.in(author for correspondence),veda@ece.iisc,ernet.in, aps@ece.iisc.ernet.in
}

\begin{abstract}
A new divide-and-conquer scheme has been proposed with Adaptive Modulation in CDMA system to improve the spectrum efficiency of the wireless communication system. The proposed method results in increase in average throughput achieved and simultaneously decrease in the outage probability of the system when the user density is high. The proposed scheme is compared with the existing algorithms and the advantage of the scheme is evident from the results obtained.

Keywords- Adaptive modulation, CDMA, TD-CDMA, arrival rate, throughput, outage probability
\end{abstract}

\section{INTRODUCTION}

One of the limitations of adaptive modulation is that the range of $C / I$ over which modulation can be changed is limited. For a given quality of service, define the dynamic range of $C / I$ in adaptive modulation as the difference in the highest and lowest thresholds of all available modulation schemes. Thus, if the set of all possible modulations used contains $M=2$ to $M=256$ QAM, then, dynamic range is the difference in thresholds of $M=256$ and $M=2$, which is about $15 \mathrm{~dB}$ for probability of error $P_{b}=10^{-3}$ in an AWGN channel [1]. Modulation schemes can adapt to the channel only if their $C / I$ falls in the dynamic range. Thus it is desirable to have low variance in $C / I$. In CDMA systems if the received signal strength is more it increases the interference to other users and if it is less, it results in outage. To restrict the resulting $C / I$, in dynamic range, power control schemes are widely used in CDMA systems.

\section{System ASSUMPTIONS}

The system considered consists of a single isolated cell of radius $R$ with a centrally located base-station. Reverse link is considered for the analysis. It is assumed that calls arrive at the base station with an arrival rate of $\lambda$ calls/sec. The mean holding time is assumed to be $1 / \mu \mathrm{sec}$. The number of active calls is a Poisson random variable with mean $\lambda / \mu$ [2], [3]. Users are assumed to be uniformly distributed in a circular cell of radius $R$. Furthermore, it is assumed that each user is gated on with a probability $\rho$ and off with probability $1-\rho$ [3]. The Signal strength received at the base station is a function of the power transmitted from the mobile, path loss, shadowing and fading effects suffered by the signal [4]. In what follows, the effect of each of these parameters is discussed in detail.

It is assumed that the power transmitted by each mobile $\left(P_{T}\right)$ is normalized to 1 watt. It is assumed that the average path loss is [5]

$$
\overline{P_{L}}=\left\{\begin{array}{cc}
\left(\frac{k}{\beta}\right)^{\alpha}, & r<\beta ; \\
\left(\frac{k}{r}\right)^{\alpha}, & r \geq \beta .
\end{array}\right.
$$

Here $r$ is uniformly distributed distance between transmitter and receiver and $k$ is a constant which depends on the maximum power received at the edge of the cell. $\alpha$ is path loss exponent. The parameter $\beta$ is included to take care of the fact that $\lim _{r \rightarrow 0} r^{-\alpha}=\infty$. The pdf of $\overline{P_{L}}$ is given in [6]

Shadowing loss is given by $S_{L}$ and the average shadowing loss by $\overline{S_{L}}$.

$$
\zeta:=10 \log _{10} S_{L} \sim N(\bar{\zeta}, \sigma)
$$

where $\bar{\zeta}=E[\zeta]$, and $\sigma$ is the variance of the normal distribution.

High vehicular velocity environment is considered. Fading loss is denoted by $F_{L}$. Thus if, $F_{e}$ denotes the fading envelop then we assume

$$
E\left(F_{e}^{2}\right)=1
$$

which means that adaptive modulation need only respond to log-normal shadowing and path-loss.

It is assumed that $\beta=0.1 R$ and $\alpha=4$. Cell size is designed on the basis of total average bit energy-to-background noise ratio $\left(\frac{k}{R_{a}}\right)^{\alpha}$ at distance $\mathrm{R}$, which occurs when a mobile is on the cell fringe. it is assumed that $\bar{\zeta} \approx 2.5 \mathrm{~dB}$ and standard deviation $\sigma \approx 7 \mathrm{~dB}$. Consider a CDMA system with $K u$ users, uniformly distributed over a cell of radius $R$. Let, $r_{1}, r_{2}, \ldots r_{k u}$ denote their distances from the base station. Let, $\zeta_{1}, \zeta_{2}, \ldots, \zeta_{k u}$ be their corresponding shadowing loss factors. They are assumed to be normally distributed random variables. The received signal strength of the $j_{\text {th }}$ mobile at the base station is denoted by $\psi_{j}$ and is defined as [4]

$$
\psi_{j}:=10^{-\zeta_{j} / 10} r_{j}^{-\alpha} \text {. }
$$

Using our model, $C / I$ of $i_{t h}$ user can be written as [7]

$$
\left(\frac{C}{I}\right)_{i}=\frac{\psi_{i}}{\sum_{\substack{\mathrm{j}=1 \\ j \neq i}}^{K_{u}} \psi_{j}+N_{O} W} \cdot \frac{W}{R}
$$

where $N_{O}$ is the noise spectral density and $W$ is the bandwidth of the system and $R$ is the bandwidth of the information with $W \geq R$. The SNR for the $j^{\text {th }}$ mobile is given by

$$
S N R_{j}=\psi_{j} / N_{O}
$$

\section{INSIGHTS INTO SYSTEM BEHAVIOR}

A number of useful insights can be obtained by carefully studying the system behavior under various conditions. Such insights will also lead naturally to the divide and conquer approach proposed by us.

\section{III-A Near-Far Effect and number of users}

Denominator in Eq. (5) shows that user with strong signal strength will suppress users with weak signal strength. This effect is called the near-far effect. To avoid this, power control is used in CDMA systems. Similary, it can be observed that more the number of users in the system lesser would be the average $(C / I)$ of the system. 
III-B Effect of Variance of Received Signal Strength

Eq. (5) can be rewritten as

$$
\begin{gathered}
\left(\frac{C}{I}\right)_{i}=X_{i}^{\prime}-Y_{i}^{\prime} \\
\text { where } \quad X_{i}^{\prime}:=\log \left(\psi_{i} \cdot W\right) \\
\text { and } \quad Y_{i}^{\prime}:=\log \left(\Gamma\left(i, K_{u}\right)+N_{O} W \cdot R .\right)
\end{gathered}
$$

Clearly $X_{i}^{\prime}$ and $Y_{i}^{\prime}$ are random variables and hence $\left(\frac{C}{I}\right)_{i}$ is also a random variable. Furthermore, since $\psi_{i}$ 's are independent random variables, $X_{i}$ 's are independent random variables. Similarly $Y_{i}$ 's are also independent random variables. Besides, $X_{i}$ and $Y_{i}$ are independent with respect to each other and hence $X_{i}^{\prime}$ and $Y_{i}^{\prime}$ are also independent with respect to each other. Thus,

$$
\operatorname{Var}\left(\left(\frac{C}{I}\right)_{i}\right)=\operatorname{Var}\left(X_{i}^{\prime}\right)+\operatorname{Var}\left(Y_{i}^{\prime}\right)
$$

Clearly, by Eq. (10) the system with the smaller variance in received signal strength also has a smaller variance in $\left(\frac{C}{I}\right)$. Such a system is preferable, based on the discussions in Section I.

III-C Outage Probability

Outage probability of a CDMA system is defined as

$$
P_{\text {outage }}=\operatorname{Pr}\left(\left(\frac{C}{I}\right)_{i} \leq T h_{\text {min }}\right)
$$

Thus if the average $C / I$ of a system is high, the corresponding outage probability decreases.

\section{PRoposed Scheme}

A divide and conquer approach with adaptive modulation in CDMA systems is proposed. Cumulative distribution function of a random variable gives a good estimate of its variance [8]. let's denote the cumulative distribution function of received SNR at the base station by $F_{S N R}$ and define it as

$$
F_{S N R}(y)=\operatorname{Pr}(S N R \leq y) \text {. }
$$

Using the typical values mentioned earlier, the received SNR at the base-station vs. $F_{S N R}$ is plotted in Fig. (1).

$I V$-A Definition of a Group

Central to the proposed scheme is the concept of a group. Divide the users into groups based on the cumulative distribution function of the received signal strength. Let there be $K_{u}$ users in the group and $\left\{x_{i}, \quad i=1\right.$ to $\left.K_{u}\right\}$ be SNRs of $K_{u}$ users. The $n_{t h}$ group is defined as follows :

$$
G r_{n}=\left\{i: \frac{n-1}{N} \leq F_{S N R}\left(x_{i}\right)<\frac{n}{N}\right\}
$$

An alternate definition is

$$
\begin{aligned}
& G r_{n}=\left\{i: l_{n-1} \leq x_{i} \leq l_{n}\right\} \\
& \text { where } l_{n-1}=F_{S N R}^{-1}\left(\frac{n-1}{N}\right) \\
& l_{n}=F_{S N R}^{-1}\left(\frac{n}{N}\right) .
\end{aligned}
$$

This is illustrated in Fig. (1). For a given division of the $x$ axis, all those users who are transmitting power in this division constitute a group. By definition of the groups the probability of number of arrivals in each group and hence expected number of users in each group is equal.

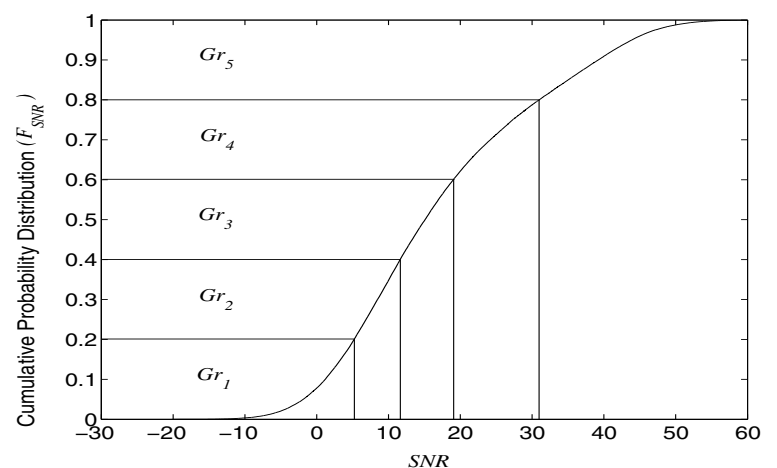

Fig. 1. Cumulative distribution function of the received signal and definition of group

\section{IV-B Orthogonal Groups}

Now the key idea of our scheme is that users of one group should not interfere with users in other groups, that is, groups should be orthogonal to each other. This can be accomplished by putting different groups on different time slots as is done in TD-CDMA systems. Thus each time slot supports many users by doing CDMA. Users in each group are orthogonal to users in other groups.

On first glance it looks like this scheme will not be advantageous as putting on time slots will reduce the transmission rate. But, it is shown that the reduction in throughput is compensated by using adaptive modulation and the grouping concept explained earlier.

Consider a TDMA system with $K$ slots of a frame. The throughput $T h$ of this system transmitting $N M$-ary symbols in each slot of duration $T_{b u}$ is given by

$$
T h=\frac{N \log _{2}(M)}{K T_{b u}}
$$

The bit rate $B$ is defined as

$$
B=\log _{2}(M)
$$

and denote the average bit rate by $\bar{B}$. Thus, the average throughput denoted by $\overline{T h}$ is given by

$$
\overline{T h}=\frac{N \bar{B}}{K T_{b u}} .
$$

It can be seen that by putting groups on time slots, transmission rate and hence throughput decreases by a factor of $K(=$ the number of groups in the system).

In adaptive modulation using 2 to $\mathrm{M}$ symbols in a constellation, average number of bits per second $\bar{B}$ is given by

$$
\begin{gathered}
\bar{B}=1 \cdot \int_{T h_{1}}^{T h_{2}} f_{C / I}(x) d x+2 \cdot \int_{T h_{2}}^{T h_{3}} f_{C / I}(x) d x \\
\quad \ldots+\log _{2}(M) \cdot \int_{T h_{\log _{2}(M)}}^{\infty} f_{C / I}(x) d x
\end{gathered}
$$

where $f_{C / I}(x)$ is the PDF of received $C / I$.

But, by dividing the users into groups, following goals are accomplished, 
- The number of users per CDMA system decreases. This results in increase in the average $C / I$ of the system as discussed earlier.

- Variance of received signal strength per user also decreases. Variance of a group is given by

$$
\operatorname{Var}\left(S N R_{G r_{n}}\right)=\int_{l_{n-1}}^{l_{n}}\left(x-m_{G r_{n}}\right)^{2} f_{S N R}(x) d x
$$

$m_{G r_{n}}$ is the mean of SNR in $n_{t h}$ group $G r_{n}$ and can be defined as

$$
m_{G r_{n}}=\int_{l_{n-1}}^{l_{n}} x f_{S N R}(x) d x
$$

Where, $f_{S N R}(x)$ is the probability density function of received SNR. By definition of a group, the difference $l_{n}-l_{n-1}$ varies for different values of $n$ and it decreases with increase in number of groups in the system. Since in Eq. (19) integrand is always positive, it can be concluded that the variance reduces with decrease in difference in limits, $l_{n}-l_{n-1}$ and hence we can say that variance of received signal strength decreases with increase in number of groups. This results in increase in average $C / I$ as discussed in Section III-B.

Above two factors result in increase in average $C / I$. Since, $\bar{B}$ is a function of the PDF of $C / I$, it increases due to increase in average $C / I$ (Eq. (18)). The increase in average $C / I$ also decreases the outage probability as discussed in Section IIIC. It is observed that at high loads (high user density) the increase in average throughput due to increase in $\bar{B}$ compensates for the decrease in average throughput due to division of users into groups. As a result, the proposed scheme results in higher throughput for higher loads.

\section{ADAPTIVE MODULATION WITH POWER CONTROL -} USING QIU AND CHAWLA'S ALGORITHM

Qiu and Chawla have developed optimization algorithms by jointly optimizing the modulation and the transmission power [6].

The system considered by the authors has finite number of cells and a fixed number of independent channels assigned to the users. If, $\mathbf{G}=\left\{G_{i j}\right\}$ denote the $N \times N$ path gain matrix for $\mathrm{N}$ users and their serving base stations and $\mathbf{P}=\left\{P_{i j}\right\}$ denote the transmission power of base $j$, the $\operatorname{SINR} \gamma_{i}$ of user $i$, is equal to

$$
\gamma_{i}(P)=\frac{G_{i i} P_{i}}{\sum_{j \neq i} G_{i j} P_{j}+n_{i}}=\frac{G_{i i} P_{i}}{I_{i}}
$$

For a specific transmission power vector $\mathbf{P}$, the total throughput $T$, which is defined as the number of bits that can be successfully transmitted within each transmitted symbol by all user is given by

$$
T(\mathbf{P})=\sum_{i} \log _{2}\left(1+k \cdot \gamma_{i}(\mathbf{P})\right)
$$

where $k$ is a constant.

Authors have proposed two optimization algorithms to optimize the modulation and transmission power.
$V$-A Qiu and Chawla's algorithm applied to the system considered

In a single cell CDMA system,

$$
\gamma_{i}(\mathbf{P})=\frac{G_{i} P_{i}}{\sum_{j \neq i} G_{j} P_{j}+n_{i}}=\frac{G_{i} P_{i}}{I_{i}}
$$

\section{A. Objective I}

Set of feasible transmission power vectors is $\Omega=$ $\left\{\mathbf{P}: \mathbf{P}_{\min } \leq \mathbf{P} \leq \mathbf{P}_{\max }\right\}$. Objective function to be maximized, called objective $\mathrm{I}$, is the total throughput $T(\mathbf{P})$

$$
\max _{\mathbf{P}} T(\mathbf{P}) \quad \text { s.t. } \mathbf{P} \in \Omega
$$

From the definition of $T(\mathbf{P})$ and Eq. (23), equating $\partial T(\mathbf{P}) / \partial P_{i}$ to zero we get the following iteration,

$$
X_{i}(\mathbf{P}(n)) \equiv \frac{\gamma_{i}}{G_{i}\left(1+k \gamma_{i}\right) \sum_{j \neq i} \frac{\gamma_{j}}{\left(1+k \gamma_{j}\right) I_{j}}}
$$

Thus, the transmission power of user $i$ at the $(n+1)$ th iteration is given by

$$
P_{i}(n+1)=\left\{\begin{array}{lll}
P_{i}^{\min }, & \text { if } & X_{i}(\mathbf{P}(n))<P_{i}^{\text {min }} \\
P_{i}^{\max }, & \text { if } & X_{i}(\mathbf{P}(n))>P_{i}^{\max } \\
X_{i}(\mathbf{P}(n)), & \text { otherwise } & \cdot
\end{array}\right.
$$

\section{B. Objective II}

In order to avoid the possibility of converging to a highly suboptimal solution authors have proposed a slightly different objective function

$$
(\mathbf{P})=\prod_{i} \gamma_{i}(\mathbf{P})
$$

The maximization problem is

$$
\max _{\mathbf{P}} O(\mathbf{P}) \quad \text { s.t. } \mathbf{P} \in \Omega
$$

From the definition of $O(\mathbf{P})$ and Eq. (23), equating $\partial O(\mathbf{P}) \partial P_{i}$ to zero, we get the following iteration

$$
Y_{i}(\mathbf{P}(n)) \equiv \frac{1}{G_{i} \sum_{j \neq i} \frac{1}{I_{j}(\mathbf{P}(n))}}
$$

The transmission power of user $i$ at the $(n+1)$ th iteration is given by

$$
P_{i}(n+1)=\left\{\begin{array}{lll}
P_{i}^{\text {min }}, & \text { if } & Y_{i}(\mathbf{P}(n))<P_{i}^{\text {min }} \\
P_{i}^{\text {max }}, & \text { if } & Y_{i}(\mathbf{P}(n))>P_{i}^{\text {max }} \\
Y_{i}(\mathbf{P}(n)), & \text { otherwise } & .
\end{array}\right.
$$

From Eq. (25) and Eq. (29) we can see that both iterations converges to same $\mathbf{P}$ for large values of $\gamma$ and in steady state throughput achieved by both algorithms is same. 
TABLE I

Simulation PARAMETERS AS PER THE SYSTEM CONSIDERED

\begin{tabular}{|c|c|c|}
\hline Parameters & Value & Comments \\
$\begin{array}{c}\text { Number of groups } \\
\text { considered }\end{array}$ & 5 & \\
$\lambda$ & & $\begin{array}{c}\text { exponentially } \\
\text { distributed }\end{array}$ \\
$1 / \mu$ & 6 minutes & \\
$\rho$ & 0.4 & \\
Channel : & & \\
$N_{O}$ & $10^{-11}$ watts $/ H z$ & log-normal \\
Shadowing & & \\
mean & & \\
variance & $2.5 \mathrm{~dB}$ & \\
$\eta$ & 4 & \\
$\beta$ & 0.1 & \\
Path-Loss & $500 \mathrm{~m}$ & \\
r, user's location & & \\
k & & \\
$P_{\text {max }}$ & & \\
Qiu and Chawlion \\
Algorithm :
\end{tabular}

TABLE II

Constellation Size, Required $C / I$ and Throughput (BITS/SYMBOL)

\begin{tabular}{|l|c|c|c|c|}
\hline M & 2 & 4 & 16 & 64 \\
\hline Thresholds & $T h_{1}$ & $T h_{2}$ & $T h_{3}$ & $T h_{4}$ \\
Min. $C / I$, in dB & 4 & 7 & 13 & 18 \\
\hline T = $\log _{2} M$ (bits/sym.) & 1 & 2 & 4 & 6 \\
\hline
\end{tabular}

\section{Simulations, Results and Discussion}

Performance results of the scheme are obtained by simulations of the scheme carried out using MATLAB. Simulation parameters for the channel considered and Qiu and Chawla's algorithm are shown in Table I. Table VI shows the thresholds for $\mathrm{Pb}=10^{-3}$ for all modulations used.

In CDMA system, $C / I$ is given by Eq. (5). It can be observed from Eq. (10) that the variance of $C / I$ depends on the variance of the received signal strength. By Dividing users in groups we ensure that a call which arrives at the base station having high signal strength does not interfere with users in other groups transmitting at lower signal strength.

Fig. (2) shows the simulated variation of variance of $C / I$ with number of groups in the system considered for 100 users in the system. It is evident that variance of received signal

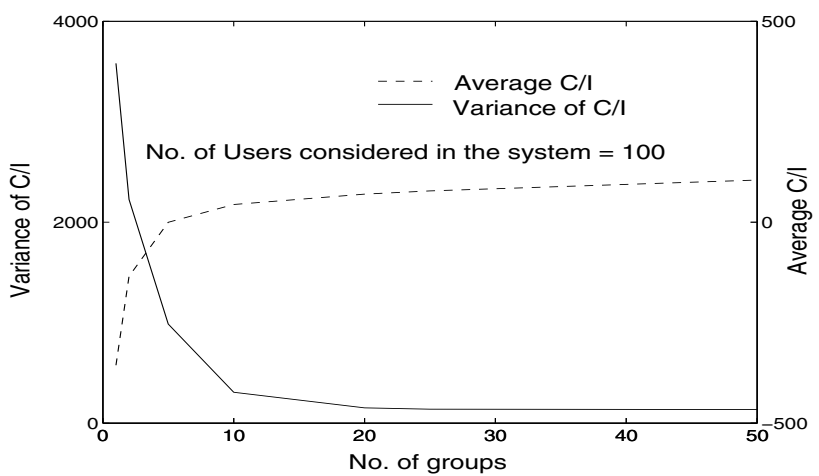

Fig. 2. Variation of the variance of $\mathrm{C} / \mathrm{I}$ and Average $\mathrm{C} / \mathrm{I}$ (in $\mathrm{dB}$ ) with no. of groups/slots

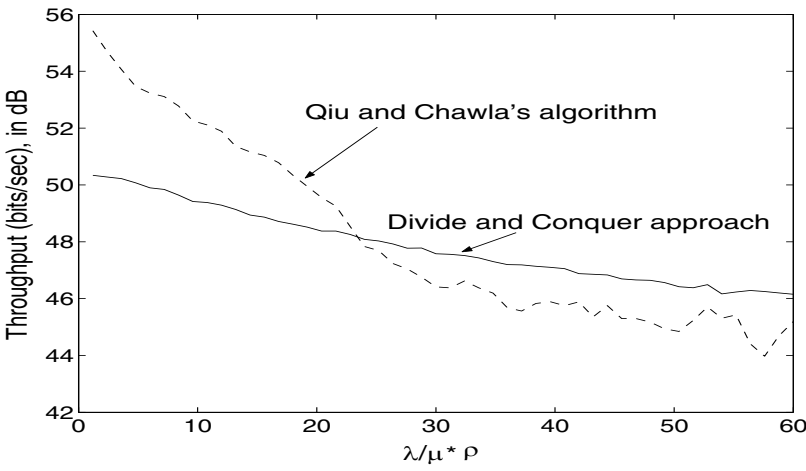

Fig. 3. Throughput comparison with Qiu's algorithm

reduces as number of group increases in the system. Thus, variance in received signal strength varies among groups. In steady state, number of users in groups remains equal. Thus, by dividing users in groups, number of interfering user to a given user decreases which results in increase in average $C / I$ of the system as it is evident from Fig. (2). Increase in average $C / I$ is exploited by adaptively changing the modulation of the system which results in gain in throughput of the system. Performance metric of adaptive modulation in communication system are average throughput and outage probability of the system. Qiu and Chawla have proposed the algorithm for implementing adaptive modulation with power control by maximizing throughput of the system. Outage probability of the system is not taken care of. As described before, both the objective functions converge to the same values of the throughput for the system considered. Fig. (3) shows the comparison of algorithm explained in Eq. (30) with the proposed method having 5 groups. The comparison is done for the varying load of the system i.e. For the average number of active users in the system which is given by $(\lambda / \mu) \rho$.

For demonstration of the concept, 5 groups are considered in the system. As can be seen from Fig. (4), Qiu and Chawla's algorithm gives a higher throughput than the scheme proposed, for the average load of the system less than 23. When load in the system increases, Qiu and Chawla's algorithm performs inferior to the scheme proposed. The reason for such variation can be attributed to the basic nature of global optimization of power control techniques. When number of users in the system is more, average $C / I$ of the system reduces and hence throughput of the system even when power control technique is used because by power control, power can be varied in a fixed range of $P_{\min }$ to $P_{\max }$. In the proposed method, number of users in a group, hence the number of interfering users on the same bandwidth is less and their signal strength has less variance as compared to the system without any grouping. These two factors, help in achieving higher throughput than adaptive modulation with power control in system subjected to high load.

As discussed earlier, Qiu and Chawla's algorithm doesn't takes care of outage probability of the system. The reason for this is the fact that objective function takes care only of the throughput of the system. Higher throughput of the system is achieved by promoting good users (high signal strength) and discouraging bad users (low signal strength). Thus it 


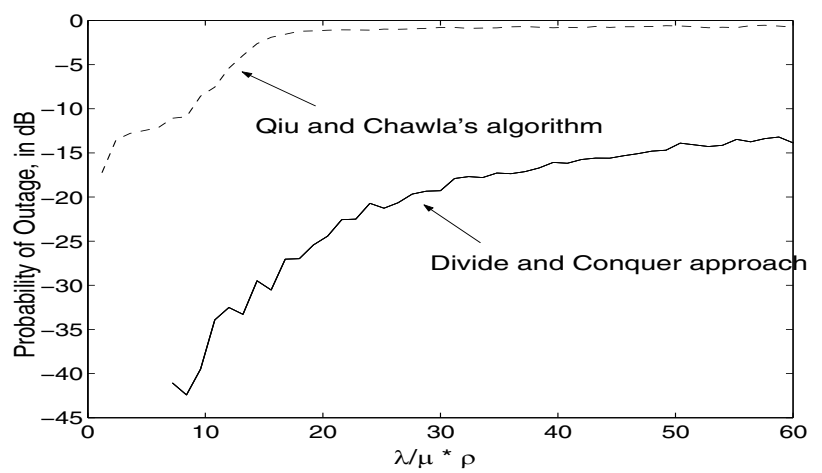

Fig. 4. Outage Probability comparison with Qiu's algorithm

doesn't result in increasing active number of users in the system. Hence, outage of the system increases. As can be seen from Fig. (4) proposed method always gives less outage probability than Qiu and Chawla's algorithm irrespective of load in the system.

It is evident from the above discussion that dividing users in groups as suggested in section IV results in increasing the throughput of the system and reduces the outage probability. Number of groups required in the system suggested is a parameter which is needed to be evaluated. More the number of orthogonal groups, more will be the slots in a time frame and which will reduce the throughput as the transmission will take place in a fraction of time alloted to the frame. Increasing slots reduces this fraction of time further. On the other hand, we get an increase in average $C / I$ of the system. More slots can be advantageous only if reduction in throughput can be compensated. As described above, we can use adaptive modulation to take advantage of the increase in $C / I$ and hence can increase the throughput of the system. As can be seen from Fig. (5), throughput of the system decreases with increase in load of the system. Having one group means all users are transmitting continuously, hence no time slots needed. As load increases, average $C / I$ of the system decreases which results in more outage and less throughput. To increase average $C / I$, number of groups should increase and by using adaptive modulation throughput of the system can be increased relatively. As evident from Fig. (5), no grouping is required if the number of active users in the group is less than 3 . If number of active users exceeds 3 , there should be two groups in the system, and if number of user is more than 40 then having 3 groups is recommended. Thus, number of groups should be increased with increase in load in the system.

Fig. (6) shows the outage probability comparison with increase in the effective load in the system. Outage probability increases with load. As evident from the figure, having more number of group results in decrease in outage probability of the system.

\section{CONCLUSION}

From the results obtained by the simulations and from the discussions in the above section it can be concluded that dividing user in orthogonal groups based on $C / I$ received from them in reverse link can prove advantageous in conditions of high load. We achieve better throughput and low outage prob-

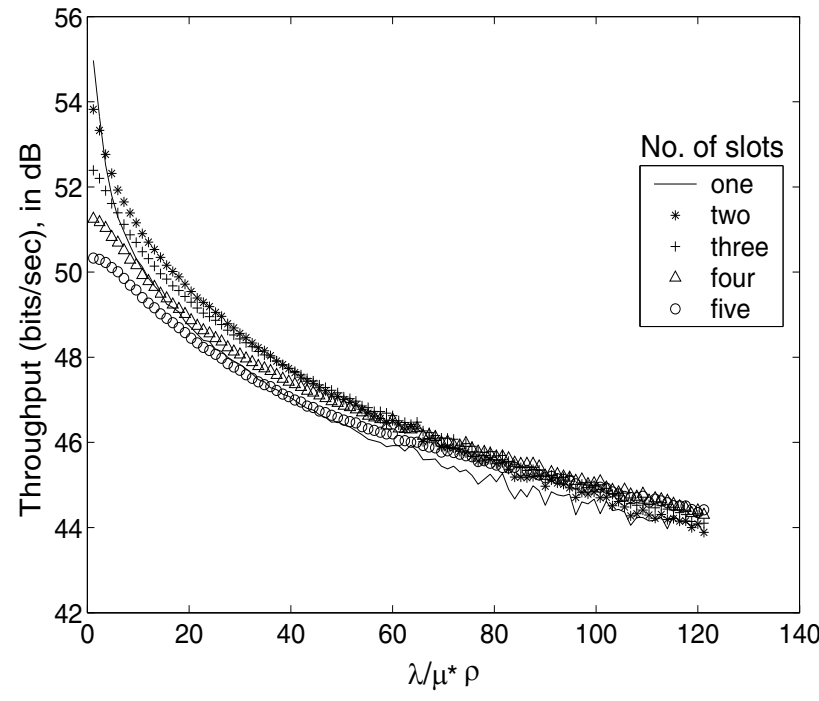

Fig. 5. Throughput comparison of different number of groups

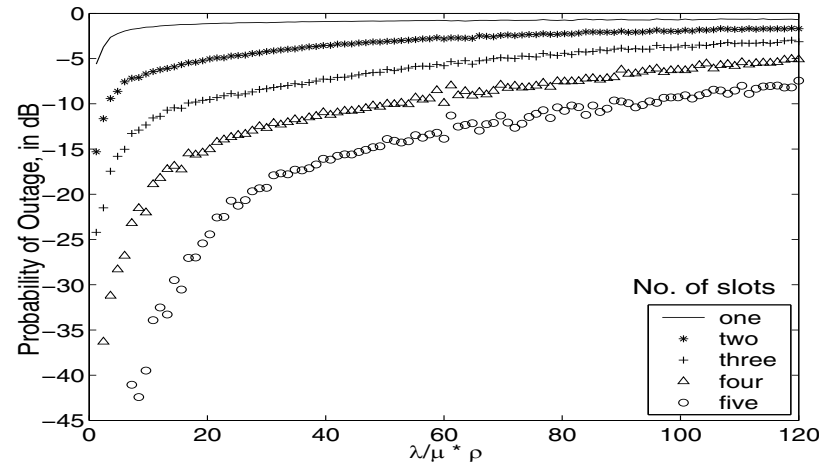

Fig. 6. Outage Probability comparison of different number of groups.

ability by the proposed method. Its by exploiting the increase in $\mathrm{C} / \mathrm{I}$ due to grouping by adaptive modulation. It is seen that the proposed method gives better throughput and less outage than the algorithm proposed by Qiu and Chawla . Number of groups in the system can be decided as per the requirements of throughput and outage probability of the system from the results obtained.

\section{REFERENCES}

[1] Toyoki Ue, Seiichi Sampei, and Norihiko Morinaga, "Symbol rate and modulation level controlled adaptive modulation/TDMA/TDD for personal communication systems," Proceedings of the IEEE VTC'95, pp. 306-310, 1995.

[2] Audrey M. Viterbi and Andrew J. Viterbi, "Erlang capacity of power controlled CDMA system," IEEE Journal in Selected Areas of Communication, vol. 11, no. 6, pp. 249-257, August 1993.

[3] D. Bertsekas and R. Gallager, Data networks, Prentice Hall, 1987.

[4] Gordon L. Stüber, Principles of Mobile Communication, Kluwer Academia Publishers, 2001

[5] Chamrœun Kchao and Gordon L. Stuber, "Analysis of a direct-sequence spread-spectrum cellular radio system,” IEEE Trans. Commun., vol. 41, no. 10, pp. 1507-1516, October 1993.

[6] Xiaoxin Qiu and Kapil Chawla, "On the performance of adaptive modulation in cellular systems," IEEE Trans. Commun., vol. 47, no. 6, pp. 884-895, June 1999.

[7] Andrew J. Viterbi, CDMA Principles of Spread Spectrum Communication, Addison-Wesley Longman, Inc., 1995.

[8] Robert M. Gray and Lee D. Davisson, An introduction to Statistical Signal Processing, http://www-isl.stanford.edu/gray/sp.html, 2001. 